\title{
Clasificación de la OMS 2004 para los tumores vesicales: resumen y comentarios
}

\author{
Álvarez Kindelán J*, Campos Hernández JP*, López Beltrán A**, Requena Tapia MJ*. \\ *Servicio de Urología, Hospital Universitario Reina Sofía, Córdoba. **Departamento de Patología Hospital \\ Universitario Reina Sofia y Facultad de Medicina Universidad de Córdoba.
}

Actas Urol Esp. 2007;31(9):978-988

\section{RESUMEN}

CLASIFICACIÓN DE LA OMS 2004 PARA LOS TUMORES VESICALES: RESUMEN Y COMENTARIOS

Los puntos clave de la última clasificación de la OMS para los tumores uroteliales no invasivos son los siguientes: la descripción de las categorías ha aumentado para mejorar su reconocimiento: los tumores con particular buen pronóstico (neoplasia papilar urotelial de bajo potencial maligno) no deben ser etiquetados como "cáncer"; esto evita el uso de una gradación ambigua tal como grado $1 / 2$ o 2/3 (como se hacía en la clasificación de la OMS de 1973); el grupo de los tumores no invasivos de alto grado es bastante grande pues contiene todos los tumores con propiedades biológicas similares a las de los carcinomas invasivos, e igualmente un alto nivel de inestabilidad genética. Este esquema es el que se propone para sustituir a la clasificación de la OMS de 1973, pero no debe invalidarse definitivamente, y usarse conjuntamente con la nueva clasificación de 2004 hasta que esta última esté lo suficientemente validada.

Palabras clave: Cáncer vesical. Patología. Genética.

\section{ABSTRACT}

THE 2004 WHO CLASSIFICATION OF BLADDER TUMORS: A SUMMARY AND COMMENTARY

The Key points of the latest World Health Organization (WHO) classification of non-invasive urothelial tumors are the following: the description of the categories has been expanded to improve their recognition: a tumor with particularly good prognosis (papillary urothelial neoplasm of low malignant potential) no longer carries the label of "cancer"; it avoids the use of ambiguous grading as grade $1 / 2$ o $2 / 3$ (as done in the 1973 WHO classification); the group of non-invasive high-grade carcinoma is large enough to virtually contain all those tumors having biological properties similar to those seen in invasive urothelial carcinoma, and a similarly high level of genetic instability. This scheme is meant to replace the 1973 WHO classification, but the use of both the 1973 and the latest WHO classification is recommended until the latter is sufficiently validated.

Keywords: Bladder cancer. Pathology. Genetics.

$\mathrm{N}^{\infty}$ o se conoce completamente la historia natural de las neoplasias uroteliales no invasivas no sujetas a tratamiento. Globalmente, el 70\% de los tumores vesicales se presentan como enfermedad superficial, y el resto como músculoinvasiva. Del total de tumores superficiales, el 70\% son lesiones de estadio Ta, el 20\% T1 y un 10\% CIS. La heterogeneidad de la enfermedad se expresa en la gran variabilidad de su evolución natural, desde los tumores Ta de bajo grado, con muy baja tasa de progresión, hasta los tumores $\mathrm{T} 1$ de alto grado asociados a progresión significativa y tasas de mortalidad específicas ${ }^{1}$. Se han estudiado muchas características de los tumores uroteliales para intentar predecir su evolución: hallazgos patológicos, análisis citológico, marcadores moleculares. 
La exactitud en el estadiaje y gradación de la enfermedad son muy importantes para decidir el tratamiento óptimo.

En Diciembre de 1998 se publicó la clasificación de consenso de la OMS/ISUP (Sociedad Internacional de Uropatólogos) de las neoplasias uroteliales vesicales ${ }^{2}$, en un intento necesario por desarrollar una clasificación aceptable y efectiva que permitiera su uso común entre urólogos, patólogos y oncólogos, clasificación que comprendía no sólo las condiciones neoplásicas, sino también las lesiones preneoplásicas.

Desde el punto de vista morfológico se identifican dos categorías diagnósticas básicas según el patrón de crecimiento de las lesiones intrapiteliales: planas y papilares. Su comportamiento clínico se relaciona con el grado de alteraciones en la arquitectura y en la citología del urotelio ${ }^{3,4}$. La última clasificación de la OMS para los tumores uroteliales no invasivos ${ }^{5}$ es idéntica a la clasificación de la OMS/ISUP de 1998 y ha sido adoptada en Pathology and Genetics of Tumours of the Urinary System and Male Genital Organs, uno de los "Libros Azules" de la nueva serie de Clasificaciones de Tumores de la OMS. El contenido del libro refleja la visión de un grupo de trabajo compuesto de uropatólogos que convinieron en la preparación de una Conferencia de Consenso realizada en Lyon, Francia en Diciembre de 2002.

\section{LESIONES INTRAEPITELIALES PLANAS}

El urotelio, el tipo de epitelio dominante que recubre la vejiga, el uréter y la pelvis renal, tiene características únicas que lo diferencian de cualquier otro tipo de epitelio. Es un epitelio de múltiples capas que contiene de forma típica muescas nucleares longitudinales en algunas de las células que forman una gran superficie celular conocida como células paraguas. Las células paraguas pueden tener alguna atipia nuclear que no debe ser confundida con displasia. La superficie de las células paraguas está formada por una membrana rígida trilaminar única compuesta por una familia de proteínas específica llamadas uroplaquinas. Dada la naturaleza única de esta mucosa, el término urotelial debe sustituir al transicional. Muchos patólogos abusan del término displasia leve para aquellas lesiones planas con citología normal y un mínimo desorden arquitectónico. Algunos caprichos en la tinción y en la fijación también pueden provocar hipercromasia en núcleos benignos. Las lesiones planas con citología normal y mínimo desorden no deberían designarse como displasia leve y ser consideradas normales ${ }^{3}$.

La clasificación de la OMS 2004 de las lesiones planas incluye la hiperplasia urotelial, la atipia urotelial reactiva, la atipia de significación desconocida, la displasia y el carcinoma in situ $^{5}$ (Tabla 1). Esta clasificación es muy similar a la publicada recientemente por López-Beltrán et al. ${ }^{6,7}$.

Tabla 1. Clasificación de la OMS 2004 de la neoplasia uroteliales no invasivas e invasivas.

Neoplasia urotelial no invasiva
Hiperplasia (plana y papilar)
Atipia reactiva
Atipia de significación desconocida
Displasia urotelial (neoplasia intraurotelial de bajo
grado)
Carcinoma urotelial in situ (neoplasia intraurotelial de
alto grado)
Papiloma urotelial
Papiloma urotelial tipo invertido
Neoplasia papilar urotelial de bajo potencial maligno
Carcinoma papilar urotelial no invasivo de bajo grado
Carcinoma papilar urotelial no invasivo de alto grado
Neoplasia urotelial invasiva
Invasión de lámina propia
Invasión muscular propia (músculo detrusor)

\section{Hiperplasia urotelial, atipia urotelial reactiva, atipia de significación desconocida}

La hiperplasia urotelial se define como la mucosa marcadamente engrosada sin atipia citológica. La hiperplasia urotelial plana ha sido tradicionalmente definida como un urotelio con mas de 7 capas celulares de espesor. En la práctica, el contaje del número de capas celulares no es reproducible. Esta lesión se suele ver adyacente a los tumores papilares uroteliales de bajo grado. Si se presenta aisladamente, no hay evidencias de que se trate de una lesión con potencial premaligno ${ }^{7}$. Sin embargo, el análisis molecular ha mostrado que la lesión, finalmente en pacientes con cáncer de vejiga, se relaciona clonalmente 
con los tumores papilares ${ }^{8}$. Las alteraciones moleculares también se observan en urotelio de apariencia histológica normal de pacientes con cáncer ${ }^{9}$.

Dentro del espectro de la hiperplasia la arquitectura papilar puede estar presente (hiperplasia papilar urotelial). Esta lesión normalmente es asintomática y generalmente se encuentra de rutina en el seguimiento cistoscópico de neoplasias papilares uroteliales ${ }^{5,8}$. Está caracterizada por un ligero campo ondulante, o crecimiento papilar cubierto por urotelio de espesor variable, faltando la atipia. La lesión, con frecuencia, tiene uno o varios pequeños capilares dilatados en su base, pero falta un tallo fibrovascular bien desarrollado. El diagnóstico de novo de una hiperplasia papilar urotelial no coloca necesariamente al paciente en riesgo para desarrollar tumores papilares, pero se recomienda su seguimiento. En los pacientes con historia de tumor papilar urotelial, esta lesión se puede asociar con un aumento en el riesgo de recidiva tumoral.

La atipia urotelial reactiva presenta cambios nucleares claramente atribuibles a un proceso reactivo/regenerativo, que ocurre en el urotelio inflamado de forma aguda o crónica. Las células son uniformemente grandes con un núcleo prominente y a veces con la cromatina vesicular. La actividad mitótica puede estar presente pero sin formas atípicas. La inflamación puede estar presente en el urotelio o en el tejido conectivo subepitelial (lámina propia). Es frecuente la historia de instrumentación uretral y litiasis.

La atipia de significación desconocida es una categoría descriptiva (no una entidad diagnóstica) de casos en los que la severidad de la atipia reactiva es desproporcionada a la extensión de la inflamación de forma que no se puede excluir definitivamente la displasia ${ }^{5}$. Los pacientes deben ser sometidos a seguimiento y reevaluados una vez ha remitido la inflamación. El uso del término atipia de significación incierta ${ }^{2}$ fue puesto en controversia por López-Beltrán et al. ${ }^{7}$ dada la impresión de que no añadía ningún valor en la práctica.

\section{Displasia urotelial}

La displasia es una lesión intraurotelial con cambios apreciables arquitectónicos y citológicos sugerentes de ser preneoplásicos pero sin criterios de carcinoma in situ (CIS). La morfología de la displasia generalmente muestra células cohesivas caracterizadas por leves cambios nucleares/nucleolares que focalmente incluye un apiñamiento nuclear irregular y ligera hipercromasia. Los nucleolos pueden ser prominentes y las figuras mitóticas, si están presentes, están localizadas en la capa basal. Las células paraguas suelen estar presentes. La mayoría de las anomalías celulares se restringen a las capas basal e intermedias pudiendo haber un aumento en el número de capas celulares. Los hallazgos nucleares y arquitectónicos son considerados mayoritariamente entre atipia reactiva y displasia ${ }^{7}$. La citoqueratina 20 puede tener valor si está presente $^{10}$ Las alteraciones de p53 y pérdidas alélicas, fundamentalmente en el cromosoma 9, también se han demostrado ${ }^{10,11}$.

Las lesiones displásicas se ven típicamente en vejigas con neoplasia urotelial y no son frecuentes en su ausencia. Dados los problemas de su reproducibilidad interobservador, la falta de una definición uniforme, y confusas publicaciones en la literatura en las que con frecuencia se combinan la displasia moderada y severa (la última actualmente considerada CIS), la historia natural de la displasia vesical no está bien establecida. La displasia es mas relevante en las neoplasia papilares no invasivas, donde su presencia indica inestabilidad urotelial y es un marcador de recidiva o progresión. La displasia de novo progresa a neoplasia vesical en un 5-19\% de los $\operatorname{casos}^{12}$.

\section{Carcinoma urotelial in situ}

El carcinoma in situ (CIS) es una lesión plana, no papilar en la que la superficie epitelial contiene células citológicamente malignas ${ }^{6,7}$. El diagnóstico morfológico de CIS requiere la presencia de atipia citológica severa (anaplasia nuclear). No son precisos cambios en todo el espesor aunque suelen estar presentes, con prominente desorden celular, con pérdida de la polaridad y la cohesividad. Las células tumorales tienden a ser grandes y pleomórficas, con un citoplasma abundante, aunque algunas son pequeñas con un alto ratio nucleocitoplásmico ${ }^{6,7,13}$. Los nucleolos son grandes y prominentes en algunas células y pueden 
ser múltiples. Las figuras mitóticas también se ven en las capas superficiales del epitelio ${ }^{14,15}$. Las células paraguas pueden estar presentes. $\mathrm{El}$ CIS no debe ser subclasificado por el grado, ya que por definición, todo CIS es una lesión de alto grado. Cuando se evalúa el grado de atipia citológica, es importante comparar las células en cuestión con el urotelio normal que lo rodea. El CIS con frecuencia es infradiagnosticado, y en el pasado era considerado con frecuencia como displasia moderada debido a que no era bien reconocido en base a la no afectación de todo el espesor epitelial por la atipia citológica. El CIS puede ser focal o difuso.

La citoqueratina 20 está anormalmente expresada en el CIS. La expresión anormal de p53 y pRB se puede correlacionar con la progresión del CIS o con la respuesta a la terapia con $\mathrm{BCG}^{10,16}$. La proteína de la matriz nuclear (NMP22) está presente. Desde el punto de vista citogenética el CIS muestra similitudes fuertes con los tumores invasivos $^{17,18}$.

El CIS primario (de novo) representa el 1-3\% de las neoplasias uroteliales y asienta fundamentalmente en la vejiga. La afectación distal ureteral oscila entre el 6-60\%, la uretra prostática en el 20-67\%, y en los ductos prostáticos y acinis en mas del $40 \%{ }^{7}$. Los pacientes con CIS se suelen presentar con clínica irritativa o hematuria, aunque también pueden permanecer asintomáticos. La exploración cistoscópica puede mostrar una mucosa normal, o eritematosa y edematosa.

El CIS primario parece progresar menos a enfermedad invasiva que el CIS secundario ${ }^{7}$. Los pacientes con CIS y tumores invasivos concomitantes fallecen en el 45-65\% de casos comparado con el $7-15 \%$ de casos de pacientes con CIS y tumor papilar concomitante. El CIS que presenta múltiples líneas celulares aneuplóides parece tener un mayor riesgo de progresión.

\section{LESIONES UROTELIALES PAPILARES NO INVASIVAS}

Este grupo comprende al papiloma, papiloma invertido, neoplasia papilar urotelial de bajo potencial maligno (PUNLMP), y el carcinoma urotelial papilar no invasivo de bajo y alto grado ${ }^{9}$ (Tabla 2).

\section{Papiloma urotelial}

El papiloma urotelial es una neoplasia exofitica benigna compuesta de un delicado tallo fibro-

Tabla 2. Hallazgos histológicos de las lesiones papilares uroteliales

\begin{tabular}{|c|c|c|c|c|}
\hline & Papiloma & PUNLMP & Cáncer papilar bajo grado & Cáncer papilar alto grado \\
\hline \multicolumn{5}{|l|}{ Arquitectura } \\
\hline $\begin{array}{l}\text { Organización } \\
\text { celular }\end{array}$ & Idéntica a lo normal & $\begin{array}{l}\text { Polaridad normal. } \\
\text { Espesor celular, } \\
\text { cohesivo }\end{array}$ & $\begin{array}{l}\text { Orden aparente, minimo } \\
\text { apiñamiento, minima falta } \\
\text { polaridad. Cohesivo }\end{array}$ & $\begin{array}{l}\text { Desorden fundamental } \\
\text { pérdida polaridad. No } \\
\text { cohesivo }\end{array}$ \\
\hline \multicolumn{5}{|l|}{ Citologia } \\
\hline Tamaño nuclear & Idéntico al normal & $\begin{array}{l}\text { Aumentados } \\
\text { uniformemente }\end{array}$ & $\begin{array}{l}\text { Aumentados con } \\
\text { variaciones en tamaño }\end{array}$ & $\begin{array}{l}\text { Aumentados con } \\
\text { variaciones en tamaño }\end{array}$ \\
\hline $\begin{array}{l}\text { Superficie } \\
\text { nuclear }\end{array}$ & Idéntico alnormal & $\begin{array}{l}\text { Aumentada, redonda- } \\
\text { oval, uniforme }\end{array}$ & $\begin{array}{l}\text { Redonda-oval. Finas } \\
\text { variaciones en contorno }\end{array}$ & Pleomorfismo marcado \\
\hline Nucleolos & Ausentes & $\begin{array}{l}\text { Ausentes } \\
\text { inaparentes }\end{array}$ & Normalmente inaparentes & Prominentes y múltiples \\
\hline Mitosis & Ausentes & Raras, basales & $\begin{array}{l}\text { Ocasionalmente cualquier } \\
\text { nivel }\end{array}$ & $\begin{array}{l}\text { Frecuentes a cualquier } \\
\text { nivel }\end{array}$ \\
\hline $\begin{array}{l}\text { Células } \\
\text { paraguas }\end{array}$ & $\begin{array}{l}\text { Presentes } \\
\text { uniformemente }\end{array}$ & Presentes & Normalmente presentes & Pueden estar ausentes \\
\hline
\end{tabular}


vascular recubierto de un urotelio de aspecto normal $^{19}$. Las células superficiales son con frecuencia prominentes, las mitosis son raras y, si están presentes, se localizan en la capa basal. El estroma puede mostrar edema o células inflamatorias. Se aplica el término de difuso cuando existe una afectación extensa de la papilomatosis. Los papilomas son tumores diplóides con baja proliferación, expresión ausente de p53, y frecuente mutación (75\%) de FGFR3 ${ }^{20}$. La expresión de la citoqueratina 20 se limita a las células superficiales (paraguas).

La incidencia del papiloma es menor del $1 \%$ de todos los tumores vesicales con una proporción hombre-mujer de $1,9 / 1^{19}$. La hematuria es frecuente. La mayoría son lesiones únicas que ocurren en pacientes jóvenes (edad media de 46 años), próximos a los meatos ureterales ${ }^{19}$. Pueden recidivar, sin embargo no progresan ${ }^{3,5}$.

\section{Papiloma invertido}

El papiloma invertido es un tumor urotelial benigno que tiene un patrón de crecimiento invertido con mínima atipia citológica celular ${ }^{21}$. Aunque no podemos hablar estrictamente de una lesión papilar, se incluye aquí porque presenta ciertos hallazgos similares al papiloma urotelial exofítico. La mayoría de los casos son lesiones polipoides solitarias, menores de $3 \mathrm{~cm}$, presentes en el trígono vesical, aunque también se pueden observar en uréter, pelvis renal o uretra $^{21}$. Histológicamente, el papiloma invertido tiene una superficie lisa cubierta por urotelio normal, con unos cordones endofíticos de células uroteliales invaginadas de forma extensa desde la superficie urotelial hasta la lámina propia subyacente sin alcanzar la pared muscular. Se han publicado casos con focos de metaplasia escamosa y diferenciación neuroendocrina. Puede haber focos pequeños de atipia citológica siendo raras las figuras mitóticas. Cuando se resecan completamente, tienen muy bajo riesgo de recidiva. En un pequeño porcentaje de casos se puede asociar con carcinoma urotelial de forma concurrente o subsiguiente. Raramente hay casos híbridos en el sentido de que la lesión presente ambos patrones de papiloma exofítico y papiloma invertido simultáneamente.
El índice hombre-mujer es de 4-5/1, la edad es muy variable (10-94 años), siendo la clínica principal la hematuria o la sintomatología obstructiva miccional. La recidiva de la enfermedad es inferior al $1 \%$ de $\operatorname{casos}^{21}$.

\section{Neoplasia papilar urotelial de bajo potencial maligno (PUNLMP)}

Se trata de un tumor papilar urotelial no invasivo que parece un papiloma urotelial exofitico pero que muestra un aumento en su celularidad que excede el espesor normal urotelial ${ }^{22,23}$. En la cistoscopia la mayoría de casos son solitarios, de 1-2 $\mathrm{cm}$ de diámetro, localizados en las paredes laterales o posterior, cercanos a los orificios ureterales ${ }^{23}$. Las papilas son finas y no fusionadas, compuestas por un urotelio de múltiples capas con mínima o ausente atipia citológica. La polaridad celular está preservada y existe una impresión de orden con mínimas variaciones en la arquitectura. Los núcleos están algo aumentados. Las capas basales pueden parecer empalizadas y la capa superficial está preservada. Estos hallazgos arquitectónicos y citológicos no deben ser valorados en áreas de cortes tangenciales de la neoplasia. Estos tumores son predominantemente diploides, con una baja tasa de proliferación, mutación de FGFR3 del $85 \%$ y pérdidas alélicas en el $80 \%$ de $\operatorname{casos}^{20,24,25}$.

$\mathrm{Su}$ mayor diferencia con el papiloma estriba en el mayor espesor del urotelio y/o con núcleos significativamente aumentados. El papiloma urotelial, por el contrario, no presenta alteraciones en la arquitectura ni atipia citológica. Las figuras mitóticas son raras en la neoplasia papilar urotelial de bajo potencial maligno, y limitadas a la capa basal. Esta lesión no se asocia con invasión ni metástasis, salvo en raros casos.

Es frecuente la hematuria micro o macroscópi$\mathrm{ca}^{26}$. La relación hombre-mujer es $5 / 1$ y la edad media de diagnóstico 65 años $^{22}$. Estos pacientes tienen un riesgo aumentado de recidiva de lesiones papilares que, ocasionalmente, pueden ser de mayor grado y pueden progresar. La recidiva tumoral, progresión en el estadio y mortalidad relacionada con el tumor ocurren en aproximadamente $35 \%, 4 \%$ y menos del $2 \%$ respectivamente (Tabla 3) ${ }^{24,27-32}$. Al realizar la primera cistoscopia de seguimiento, el 68\% de pacientes que están libres de tumor permanecerán libres de tumor por un periodo de al menos 5 años ${ }^{23}$. 


\section{Carcinoma urotelial de bajo grado no invasivo}

Neoplasia urotelial revestida de brotes papilares que muestra una apariencia ordenada pero con variaciones fácilmente reconocibles en la arquitectura y citología ${ }^{23,29}$. Histológicamente el tumor muestra finas papilas con ramificaciones frecuentes, mínima fusión, y variaciones en la polaridad nuclear, tamaño y cubierta nuclear, patrón de cromatina y con presencia de nucleolos. Las figuras mitóticas no son frecuentes y normalmente se ven en la mitad inferior del urotelio, aunque se pueden observar a cualquier nivel. Las secciones tangenciales próximas a la base del urotelio pueden desorientar por la presencia de un urotelio inmaduro con frecuente actividad mitótica. Un espectro de anomalías arquitectónicas y citológicas pueden coexistir dentro de una única lesión, reforzando la importancia de examinar toda la lesión aplicando el mayor grado de anormalidad. Es frecuente la expresión alterada de la citoqueratina 20, CD44, p53 y p63 $22,27,28$.

La relación hombre-mujer es de 2.9/1 y la edad media al diagnóstico de 70 años $^{31}$. La mayoría de pacientes se presentan con hematuria y tienen un tumor único en la cara posterior o laterales, aunque el $22 \%$ tienen 2 o mas tumores $^{22,23}$. La tasa de recidiva, progresión del estadio y mortalidad relacionada con el tumor son aproximadamente del 50\%, 10\% y 5\% respectivamente $^{27-29,31,32}$ (Tabla 3).

\section{Carcinoma urotelial de alto grado no invasivo}

Se trata de una neoplasia urotelial recubierta de brotes papilares que muestra un moderadomarcado patrón arquitectónico de desorden y atipia citológica. Histológicamente las papilas están

Tabla 3. Pronóstico de las lesiones papilares uroteliales

\begin{tabular}{lcccc}
\hline & Papiloma & $\begin{array}{c}\text { Neoplasia bajo } \\
\text { potencial maligno }\end{array}$ & $\begin{array}{c}\text { Carcinoma } \\
\text { bajo grado }\end{array}$ & $\begin{array}{c}\text { Carcinoma } \\
\text { alto grado }\end{array}$ \\
\hline Recidiva & $0-8 \%$ & $27-47 \%$ & $48-71 \%$ & $55-85 \%$ \\
Progresión grado & $2 \%$ & $11 \%$ & $7 \%$ & No aplicables \\
$\begin{array}{l}\text { Progresión } \\
\text { estadio }\end{array}$ & $0 \%$ & $0-4 \%$ & $2-12 \%$ & $15-40 \%$ \\
Supervivencia & $100 \%$ & $93-100 \%$ & $82-96 \%$ & $74-90 \%$ \\
\hline
\end{tabular}

con frecuencia fusionadas. Los núcleos a menudo son pleomórficos, con nucleolos prominentes y alteraciones en la polaridad. Las figuras mitóticas, con formas atípicas incluidas, se ven frecuentemente a todos los niveles del urotelio. El espesor del urotelio varía considerablemente. Dentro de esta categoría hay un espectro de atipia, el mayor de cual muestra un pleomorfismo nuclear marcado y difuso (idéntico al visto en el grado 3 de la clasificación de la OMS de 1973). Estos tumores también tienen un alto riesgo de asociación con enfermedad invasiva en la biopsia. De forma paralela al alto grado de atipia citológica dentro de estas lesiones, la mucosa urotelial plana circundante puede mostrar la presencia de CIS.

Los cambios de expresión de citoqueratina 20, p53 y p63 y la anueuploidía son mas frecuentes que en las categorías previas ${ }^{33}$. Las alteraciones moleculares en estos tumores muestran con frecuencia sobreexpresión de p53, HER2 o EGFR, y pérdidas en p21 o p27 comparables a las vistas en los tumores invasivos ${ }^{17}$. Las lesiones no invasivas de alto grado (pTaG3) son genéticamente idénticas a los tumores invasivos. Un estudio basado en hibridación genómica comparativa muestra delecciones en 2q, 5q, 10q y 18q así como ganancias en $5 p$ y $20 q^{17}$.

La hematuria es frecuente y el aspecto endoscópico varía desde formas papilares hasta tumores únicos o múltiples de aspecto nodular/sólido ${ }^{17}$. El carcinoma papilar urotelial de alto grado tiene mucho mayor riesgo de progresión que las lesiones de bajo grado, que oscila entre el $15 \%$ y el $40 \%{ }^{5}$.

\section{NEOPLASIAS UROTELIALES INVASIVAS Invasión de la lámina propia}

La invasión de la lámina propia se caracteriza por la presencia de células uroteliales neoplásicas dentro de la lámina propia en forma de nidos, racimos o como células sueltas, a menudo asociada con una marcada retracción por artefacto. Otra característica de la invasión tumoral, aunque no siempre está presente, es una respuesta del estroma de tipo inflamatorio o desmoplásico asociada a la 
neoplasia. En los carcinomas papilares de bajo grado, se puede observar dentro de la lámina propia, grandes nidos redondeados de urotelio con empalizada y rodeados por estroma de apariencia normal. Esto representa un patrón de crecimiento invertido de los carcinomas no invasivos ${ }^{3,7,9,13}$.

La retracción prominente por artefacto alrededor de los nidos tumorales infiltrantes en la lámina propia, a menudo se diagnostican como invasión vascular. Esta es poco común en los casos con invasión de lámina propia. Este diagnóstico se debe reservar para los casos inequívocos o para aquellos en los que se confirma mediante inmunohistoquímica ${ }^{34}$. En algunas resecciones transuretrales, se puede distinguir la lámina propia, reconociendo la muscularis mucosa así como los vasos de gran calibre. Esto permite el subestadiaje de los tumores que invaden la lámina propia, basado en la relación de estos tumores con la muscularis mucosa (por encima, en o por debajo). Este esquema ha mostrado ser de significación pronóstica. Los tumores invasivos se deben clasificar como de alto o bajo grado de igual forma que la usada para las lesiones no invasivas.

\section{Invasión de la muscular propia (músculo detrusor)}

La diferenciación en una resección transuretral entre la invasión de la muscularis mucosa o de la muscular propia, puede ser en ocasiones dificil ${ }^{34}$. Un tumor extensamente infiltrante en el que se observan entremezcladas ocasionales bandas de músculo, puede representar tanto la muscularis mucosa como la muscular propia rota y distorsionada. En estos casos, las tinciones especiales como la tinción de Masson o la de Actina por inmunohistoquímica pueden ayudar a identificar todo el músculo liso; la presencia de numerosas fibras de músculo liso entremezcladas con los nidos tumorales, pueden llevar a la conclusión de que se trata de invasión de muscular propia ${ }^{34}$. Los casos en que la invasión de la muscular propia es dudosa deben ser comunicados al urólogo.

\section{CUALIDADES DE LA CLASIFICACIÓN DE LA OMS DEL 2004}

1. Aceptación por un gran número de uropatólogos, permitiendo una uniformidad de terminología y definiciones.
2. Criterios detallados para distintas condiciones preneoplásicas y grados tumorales con gran reproducibilidad interobservador.

3. Coherencia razonable con la terminología usada en la citología de orina, facilitando la correlación citohistológica y de fácil uso para el urólogo en el manejo de los pacientes.

4. Creación de una nueva categoría que identifique a los tumores (PUNLMP) con un riesgo de progresión mínimo, con la que los pacientes eviten el diagnóstico de cáncer y las consecuencias tanto psicológicas como económicas (ej. seguros) que conlleva. Además que permita evitar el diagnóstico de lesión benigna (ej. Papiloma), con el que los pacientes no tendrían el seguimiento adecuado (esta propuesta sigue las indicaciones hechas por Montironi y col en $1987^{34}$.

5. Identificación de un grupo distintivo de tumores (carcinoma papilar urotelial de alto grado y CIS) que son los candidatos ideales para terapia intravesical.

6. Identificación de un gran grupo de tumores con alto riesgo de progresión que deben tener un seguimiento estrecho.

7. Evitar la ambigüedad en las categorias diagnósticas (ej. grado1/2, grado 2/3).

8. Estratificación de los tumores vesicales en categorías de significación pronóstica.

\section{CONVERSIÓN ENTRE LOS SISTEMAS DE LA OMS DE 1973 Y 2004}

En la introducción de 1998 de la clasificación de los tumores vesicales de la OMS/ISUP surgieron varios puntos de controversia, que se debieron principalmente a la falta de validación, reproducibilidad y estudios de conversión. En particular, no existía ningún esquema comparando las clasificaciones de 1973 y 1998 (2004). Básicamente los tumores de grado I (clasificación de 1973) se deben dividir en PUNLMP y carcinomas de bajo grado, mientras que la mayoría de los de grado 2 y todos los de grado 3 se definen como carcinomas de alto grado. En este esquema, los tumores de grado 2 cuya morfología limita con la de los tumores de grado 1, pasan a ser 


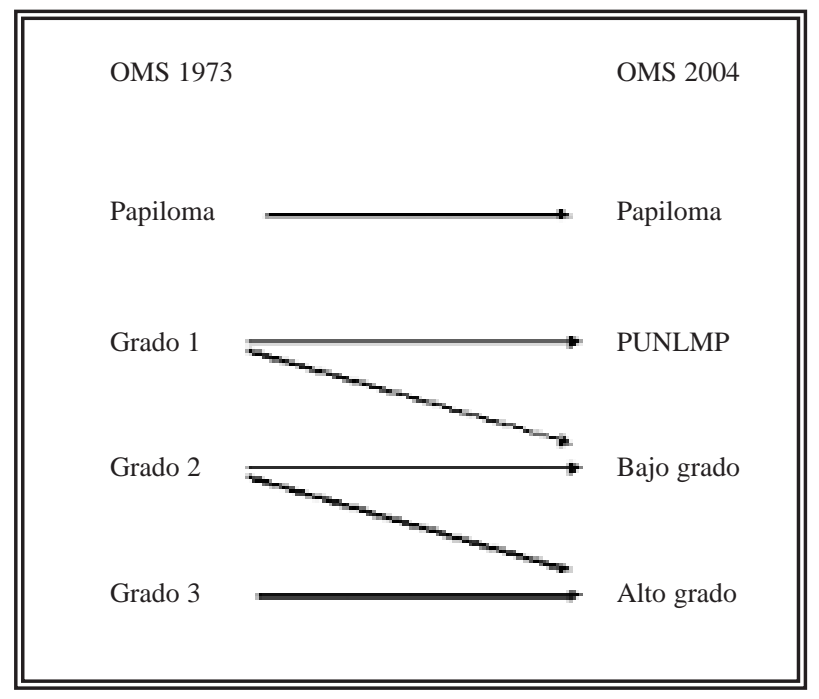

FIGURA 1. Relacion entre la clasificación OMS de 1973 y la clasificación OMS de 2004 de los tumores uroteliales papilares.

carcinomas de bajo grado ${ }^{2}$ (Fig. 1). En este punto, la clasificación de la OMS introducida en 1999 es casi idéntica a la clasificación de la OMS/ISUP, la diferencia estriba que la primera subdivide el bajo y alto grado en 3 grados (grado I, II y III) ${ }^{35,36}$.

Algunos autores 37,38 piensan que la propuesta de 1998 de la OMS/ISUP representa tan sólo un cambio en la terminología, en el sentido de que PUNLMP es equivalente al carcinoma grado 1, mientras que los carcinomas de bajo y alto grado son los equivalentes al grado 2 y grado 3 de la clasificación de 1973 de la OMS respectivamente.

\section{¿QUÉ CLASIFICACIÓN USAR?}

La cuestión de cuál es la mejor clasificación actual de las neoplasias vesicales papilares se ha discutido en varias reuniones recientes, incluida la Ancona International Consultation on the diagnosis of Non Invasive Urothelial Neoplasms (1 1-12 de Mayo de 2001). La discusión giró en torno al esquema de 1973 frente a las clasificaciones de 1998 (ahora clasificación de 2004) y 1999 de la OMS, sin alcanzar un consenso total sobre cuál debería usarse por los patólogos y seguida por los urólogos y oncólogos. Los Dres. Bostwick y Mikuz $^{37}$ representaban la opinión de la mayoría: la clasificación de la OMS de 1973 para los carcinomas papilares uroteliales era todavía superior a las demás alternativas (incluidas las de 1998 de la OMS/ISUP y 1999 de la OMS), reconociendo que era conveniente afinar más algunos criterios diagnósticos. Los Dres. C. Busch y F. Algaba ${ }^{38}$ abogaron por el papel actual de la clasificación de 1998 de la OMS/ISUP y de la de 1999 de la OMS en la práctica de la urología y la patología (opinión minoritaria).

La clasificación de 1973 es todavía preferida por algunos autores ${ }^{37}$ con pequeñas modificaciones de cara a un uso internacional que permita validar estudios de comparación de resultados entre distintos centros. Esto se debe al hecho de que la clasificación de 1973 para el estadiaje de los tumores de vejiga es un método fuerte, clínicamente probado, ampliamente usado y razonablemente reproducible para artículos de uropatología, y es la recomendada con pequeñas modificaciones.

Los cambios en la clasificación traen consigo problemas que llevan a la confusión, al menos por un tiempo. Con el objetivo de minimizar este problema, se recomienda el uso de ambas clasificaciones (1973 y 2004 de la OMS) hasta que esta última esté lo suficientemente validada.

\section{GENÉTICA MOLECULAR}

La patología molecular podría tener un papel importante en posteriores afinamientos de la clasificación. Los estudios de genética publicados han clasificado los tumores según el esquema de 1973. Ahora se necesitan nuevos estudios que vinculen la información genética disponible al esquema de 2004 de la OMS $^{17}$. Estudios actuales apuntan una relación entre dos vías genéticas con entidades definidas morfológicamente ${ }^{16,17,35,36}$ (Fig. 2). La denominada categoría genéticamente estable incluye tumores papilares no invasivos de bajo grado (pTa, G1, y G2), mientras que la categoría inestable comprende los carcinomas de alto grado (incluyendo pTa, G3 y CIS) y los carcinomas de crecimiento invasivo (estadio pT1-4). Las neoplasias de vejiga de bajo grado no invasivas tan sólo presentan algunas alteraciones en el genoma y son por tanto clasificadas como genéticamente estables ${ }^{35}$. Las neoplasias de alto grado y de crecimiento invasivo son consideradas como genéticamente inestables y tienen numerosas aberraciones cromosómicas. 


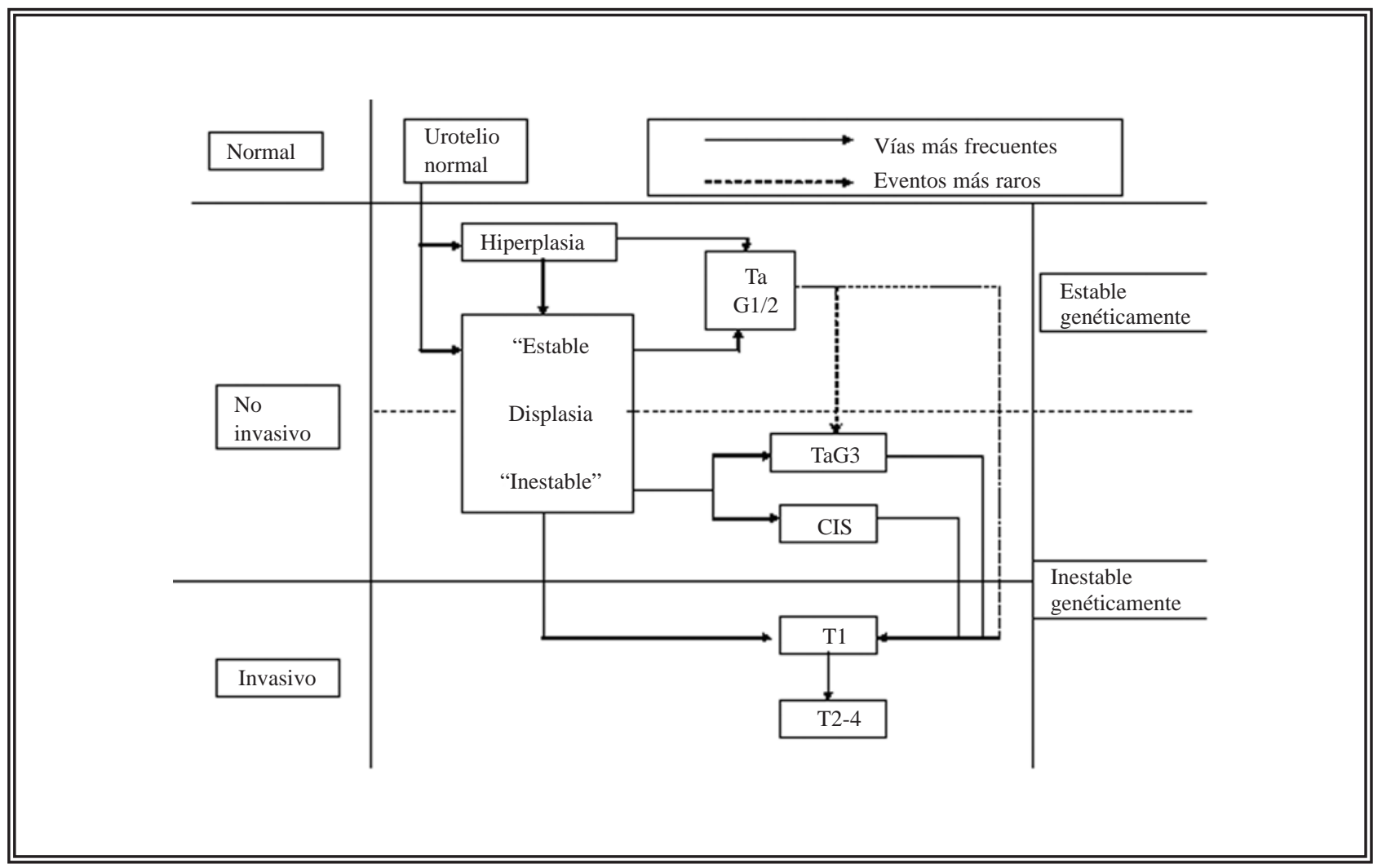

FIGURA 2. Principales vías genéticas en el desarrollo de los tumores vesicales uroteliales.

\section{CONCLUSIONES}

El uso coherente de la clasificación de la OMS del 2004 de los tumores uroteliales no invasivos, debería dar como resultado un diagnóstico uniforme de los tumores, estadiándolos de acuerdo al potencial de riesgo, y debería facilitar los estudios comparativos, la incorporación de datos moleculares y la identificación de neoplasias genéticamente inestables y agresivas.

Hasta que el sistema del 2004 esté totalmente validado desde los puntos de vista clínico y pronóstico, los tumores se deben estadiar de acuerdo a la clasificación del 2004 y de 1973 de la OMS.

La patología molecular podría jugar un papel en nuevos afinamientos del sistema de clasificación.

\section{REFERENCIAS}

1. Kirkali Z, Chan T, Manoharan M, Algaba F, Busch C, Cheng L, et al. Bladder cancer: epidemiology, staging and grading, and diagnosis. Urology. 2005;66(6 Suppl 1):434 .
2. Epstein JI, Amin MB, Reuter VR, Mostofi FK. The World Health Organization/International Society of Urological Pathology consensus classification of urothelial (transitional cell) neoplasms of the urinary bladder. Bladder Consensus Conference Committee. Am J Surg Pathol. 1998;22(12): 1435-1448.

3. Montironi R, López-Beltrán A, Mazzucchelli R, Bostwick DG. Classification and grading of the non-invasive urothelial neoplasms: Recent advances and controversies: $\mathrm{J}$ Clin Pathol. 2003;56(2):91-95.

4. Montironi R, Scarpelli M, Mazzucchelli R, Hamilton PW, Thompson D, Ranger-Moore J, et al. Subvisual changes in chromatin organization state are detected by karyometry in the histologically normal urothelium in patients with synchronous papillary carcinoma. Hum Pathol. 2003;34(9):893-901.

5. Sauter G, Algaba F, Amin MB, Busch C, Cheville J, Gasser T, et al. Noninvasive urothelial neoplasias;WHO classification of noninvasive papillary urothelial tumors. En World Health Organization classification of tumors. Pathology and genetics of tumors of the urinary system and male genital organs. Eble JN, Epstein JI, Sesterhenn I (eds): Lyon, IARCC 2004

6. Lopez-Beltran A, Luque RJ, Moreno A, Bollito E, Carmona E, Montironi R. The pagetoid variant of bladder urothelial carcinoma in situ: A clinicopathological study of 11 cases. Virchows Arch. 2002;441(2):148153. 
7. López-Beltrán A, Cheng L, Andersson L, Brausi M, de Matteis A, Montironi R, et al. Preneoplastic non-papillary lesions and conditions of the urinary bladder: an update based on the Ancona International Consultation. Virchows Arch. 2002;440(1):3-11.

8. Hartmann A, Moser K, Kriegmair M, Hofstetter A, Hofstaedter F, Knuechel R. Frequent genetic alterations in simple urothelial hyperplasias of the bladder in patients with papillary urothelial carcinoma. Am J Pathol. 1999; 154 (3):721-727.

9. Muto S, Horie S, Takahashi S, Tomita K, Kitamura T. Genetic and epigenetic alterations in normal bladder epithelium in patients with metachronous bladder cancer. Cancer Res. 2000;60(15):4021-4025.

10. Harnden P, Eardley I, Joyce AD, Southgate J. Cytokeratin 20 as an objective marker of urothelial dysplasia. Br J Urol. 1996;78(6):870-875.

11. Hartmann A, Schlake G, Zaak D, Hungerhuber E, Hofstetter A, Hofstaedter F et al. Occurrence of chromosome 9 and p53 alterations in multifocal dysplasia and carcinoma in situ of human urinary bladder. Cancer Res. 2002;62(3): 809-818.

12. Cheng L, Cheville JC, Neumann RM, Bostwick DG. Natural history of urothelial dysplasia of the bladder. Am J Surg Pathol. 1999;23(4):443-447.

13. McKenney JK, Gomez JA, Desai S, Lee MW, Amin MB. Morphologic expressions of urothelial carcinoma in situ: A detailed evaluation of its histologic patterns with emphasis on carcinoma in situ with microinvasion. Am J Surg Pathol. 2001;25(3):356-362.

14. Helpap B, Kollermann J. Assessment of basal cell status and proliferative patterns in flat and papillary urothelial lesions: A contribution to the new WHO classification of the urothelial tumors of the urinary bladder. Hum Pathol. 2000;31(6):745-750.

15. Bostwick DG, Ramnani D, Cheng L. Diagnosis and grading of bladder cancer and associated lesions. Urol Clin North Am. 1999;26(3):493-507.

16. Cordon-Cardo C, Cote RJ, Sauter G. Genetic and molecular markers of urothelial premalignancy and malignancy. Scand J Urol Nephrol. 2000;(205):82-93.

17. Simon R, Jones PA, Sidransky D, Cordon-Cardo C, Cairns P, Amin MB et al. Genetics and predictive factors of noninvasive urothelial neoplasias, WHO classification of noninvasive papillary urothelial tumors. En World Health Organization Classification of tumors. Pathology and genetics of tumors of the urinary system and male genital organs. Editores Eble JN, Epstein JLSesterhenn I;Lyon, IARCC 2004;pp 120-123.

18. Stampfer DS, Carpinito GA, Rodriguez-Villanueva J, Willsey LW, Dinney CP, Grossman HB, et al. Evaluation of NMP22 in the detection of transitional cell carcinoma of the bladder. J Urol. 1998;159(2):394-398.

19. Mckenney JK, Amin MB, Young RH. Urothelial (transitional cell) papilloma of the urinary bladder: A clinicopathologic study of 26 cases. Mod Pathol. 2003;16(7): 623-629.

20. Van Rhijn BW, Montironi R, Zwartholl EC, Jobsis AC, van der Kwast TH. Frequent FGFR3 mutations in utothelial papilloma. J Pathol. 2002;198(2):245-251.
21. Sauter G. Inverted papilloma, WHO classification of non-invasive papillary urothelial tumors. En World Health Organization Classification of tumors. Pathology and genetics of tumors of the urinary system and male genital organs. Editado por Eble JN, Epstein JI, Sesterhenn I (eds): Lyon, IARCC 2004: 114.

22. Holmang S, Andius P, Hedelin H, Wester K, Busch C, Johansson SL. Stage progression in Ta papillary urothelial tumors: Relationship to grade, immunohistochemical expression of tumor markers, mitotic frequency and DNA ploidy. J Urol. 2001;165(4):1124-1128.

23. Holmang S, Johansson SL. Stage Ta-T1 bladder cancer: the relationship between findings at first followup cystoscopy and subsequent recurrence and progression. $J$ Urol. 2002;167: 1634-1637.

24. Cheng L, Neumann RM, Bostwick DG. Papillary urothelial neoplasms of low malignant potential. Clinical and biologic implications. Cancer. 1999;86(10):2102-2108.

25. Cheng L, MacLennan GT, Zhang S, Wang M. Pan CX, Koch MO. Laser capture microdissection analysis reveals frequent allelic losses in papillary urothelial neoplasm of low malignant potential of the urinary bladder. Cancer. 2004; 101(1):183-188.

26. Fujii Y, Kawakarni S, Koga F, Nemoto T, Kihara K. Longterm outcome of bladder papillary urothelial neoplasms of low malignant potential. BJU Int. 2003;92(6):559-562.

27. Desai S, Lim SD, Jimenez RE, Chun T, Keane TE. McKenney JK, et al. Relationship of cytokeratin 20 and CD44 protein expression with WHO/ISUP grade in pTa and pT1 papillary urothelial neoplasia. Mod Pathol. 2000; 13(12):1315-1323.

28. Alsheikh A, Mohamedali Z, Jones E, Masterson J, Gilks CB. Comparison of the WHO/ISUP classification and cytokeratin 20 expression in predicting the behavior of low-grade papillary urothelial tumors. World/Health Organization/Internattional Society of Urologic Pathology. Mod Pathol. 2001;14(4):267-272.

29. Alvarez-Kindelán J, Lopez Beltran A, Anglada Curado F, Moreno Arcas P, Carazo Carazo JL, Regueiro Lopez JC, et al. Diferencias clínico-patológicas entre neoplasia vesical de bajo potencial maligno y carcinoma de bajo grado. Actas Urol Esp. 2001;25(9): 645-650.

30. Samaratunga H, Makarov DV, Epstein JL. Comparison of WHO/ISUP and WHO classification of noninvasive papillary urothelial neoplasms for risk of progression. Urology. 2002;60(2):315-319.

31. Oosterhuis JW, Schapers RF, Janssen-Heijnen ML, Pauwels RP, Newling DW. Histological grading of papillary urothelial carcinoma of the bladder: Prognostic value of the $1998 \mathrm{WHO} / \mathrm{ISUP}$ classification system and comparison with conventional grading system. J Clin Pathol. 2002;55 (12):900-905.

32. Pich A, Chiusa L, Formiconi A, Galliano D, Bortolin P, Navone R. Biologic differences between noninvasive papillary urothelial neoplasms of low malignant potencial and low grade (grade 1) papillary carcinomas of the bladder. Am J Surg Pathol. 2001;25(12):1528-1533.

33. Schmitz-Drager BJ, van Roeyen CR, Grimm MO, Gerharz CD, Decken K, Schulz WA, et al. p53 accumulation in precursor lesions and early stages of bladder cancer. World J Urol. 1994;12(2):79-83. 
34. Lopez Beltran A, Bassi P, Pavone Macaluso M, Montironi R. Handling and pathology reporting of specimens with carcinoma of the urinary bladder, ureter, and renal pelvis. Eur Urol. 2004;45(3):257-266.

35. Richter J, Jiang F, Gorog JP, Sartorius G, Egenter C, Gasser TC, et al. Marked genetic differences between stage pTa and stage pT1 papillary bladder cancer detected by comparative genomic hybridization. Cancer Res. 1997;57 (14):2860-2864.

36. Richter J, Wagner U, Schrami P, Maurer R, Alund G, Knonagel $\mathrm{H}$ et al. Chromosomal imbalances are associated with a high risk of progression in early invasive (pT1) urinary bladder cancer. Cancer Res. 1999;59(22): 5687-5691.
37. Bostwick DG, Mikuz G. Urothelial papillary (exophytic) neoplasms. Virchows Arch. 2002;441(2):109-116.

38. Busch C, Algaba F. The WHO/ISUP 1998 and WHO 1999 systems for malignancy grading of bladder cancer. Scientific foundation and translation to one another and previous systems. Virchows Arch. 2002;441(2):105-108.

Correspondencia autor: Dr. J. Álvarez Kindelán Servicio de Urología. Hospital Reina Sofía. Avda. Menéndez Pidal, s/n - 14004 Córdoba Tel: 957011057

E-mail autor: urolhapal@andaluciajunta.es Información artículo: Original 